Medical genetics

\title{
Collagen and inherited connective tissue diseases
}

\section{from Bryan Sykes}

COLlagen aficionados have long cherished the idea that abnormalities in their protein lay behind the inherited disorders of connective tissue. This complex mixture of clinical entities presents with mechanical failure of tissues like bone, gut and blood vessel walls, ligaments, tendon and skin, often with disastrous results. Since collagen is the principal stress-bearing component of the susceptible tissues their belief is reasonable but, largely owing to the complexity of the molecule, substantiation has till now been anecdotal, meagre and indirect. Obviously, collagen structural genes are the prime candidates for the mutant loci and gene probes now being used to test the hypothesis are coming up with evidence to support it. It is too early to say whether the understanding of collagen gene mutation will ever reach the levels of sophistication seen with globin but an interdisciplinary meeting* on osteogenesis imperfecta, heard the first encouraging reports that, one day, it might.

Collagen molecules consist of three collagen $\alpha$-chains wound round one another in a regular helix. At least seven distinct $\alpha$-chains have been found (labelled $\alpha 1(\mathrm{I})$ through to $\alpha 1(\mathrm{~V}), \alpha 2(\mathrm{I})$ and $\alpha 2(\mathrm{~V})$ ) and combine in different types of collagen molecules - types I and II, III being the major consitutents of connective tissues.

Fibroblasts cultured from a perinatal lethal case (Type II disease) synthesised an $\alpha 1$ (I) dimer disulphide bonded in $\alpha$, CB6 towards the carboxy terminus of the helical region (B. Steinman, UniversitatsKinderklinik, Zurich). This defect probably arose from a glycine-cysteine substitution and, since neither parent was affected nor synthesized the abnormal chain, a new dominant mutation was proposed. Any substitution for glycine in the helix would prevent perfect packing, though it was conceded during discussion. (A. Miller, University of Oxford) that the degree of distortion is unknown. To explain the severity of the mutation of a single allele it was suggested (B. Sykes, John Radcliffe Hospital, Oxford) that since each Type I molecule contained two $\alpha 1$ (I) chains the gene product of one mutant allele could theoretically be incorporated into three-quarters of the molecules. Taken together with the drastic effects of glycine substitution on helix stability this may explain the high mutation rate in many collagen diseases.

The Second International Symposium on Osteogenesi imperfecta: Brittle Bone Syndrome was held in (ixford, August 30-31, 1983
Studies on cultured fibroblasts (A. Nicholls, MRC Clinical Research Centre, London) had also led to the discovery of a patient who failed to incorporate pro- $\alpha 2$ (I) precursor chains into mature Type 1 molecules. The symptoms were surprisingly mild, presumably because compensatory formation of $[\alpha 1(\mathrm{I})]_{3}$ trimer was an effective mechanical substitute. Though pro- $\alpha 2$ (I) levels were normal, $\mathrm{S}_{1}$ mapping revealed that the patient was homozygous for a mutation at the 3 ' end of the gene corresponding to the C-propeptide region, the effect of which was to block incorporation (T. Pihlajamiena, Rutgers Medical School, New Jersey). The parents were consanguinious and heterozygosity was confirmed.

Much more perplexing was a 300 base pair deletion at the $3^{\prime}$ end of the enigmatic $\alpha$ (I) like gene found in four out of six perinatal lethal cases from widely different ethnic backgrounds (F.M. Pope, MRC Clinical Research Centre, London). This gene, localised to chromosome 12 , shares sequence homology with $\alpha \mathrm{l}(\mathrm{I})$ (chromo- some 17) but is clearly distinct from it. That it may be important in cartilage or bone formation was suggested by its strong hybridization to mRNA from a rat chondrosarcome (K.S.E. Cheah, Imperial Cancer Research Fund Laboratories, London). However, one parent in each case was heterozygous for the deletion also and one parent at least had, in retrospect, symptoms of other connective tissue disease. Irrelevant normal variants of the gene were unlikely explanations since about 400 other individuals did not have a deleted allele and the consensus was a possible compound heterozygosity.

The same gene was used to illustrate testing for causal mutations by segregation of single and multiple restriction site variants (B. Sykes) who showed how it could be excluded from causality in two pedigrees of dominantly inherited Type 1 disease.

Most delegates left the meeting optimistic that the disease loci have been identified and that the effort of many laboratories will one day be rewarded by a unified theory whereby collagen structural gene mutations will explain first the biochemistry, then the complex clinical and genetic picture of these crippling diseases.

Bryan Sykes is on the staff of the Nuffield Department of Pathology, John Radcliffe Hospital, University of Oxford, Oxford OX3 9DU.

\section{Astronomy}

\section{Structure and evolution of the Magellanic Clouds}

\section{from Sidney van den Bergh and Klaas S. de Boer}

RECENTLY over a hundred astronomers from the northern and southern hemispheres gathered to discuss the interpretation of new observations of the Magellanic Clouds*. Since the last such meeting, in 1969, understanding of the Magellanic Clouds has been revolutionized by the completion of 4-m class telescopes in the southern hemisphere (Cerro Telolo Inter-American University and European Southern Observatory telescopes in Chile, and the Anglo-Australian telescope in Australia), by the development of highly efficient panoramic detectors such as the charge coupled device, and by observations from space.

Because of their proximity the Large and Small Magellanic Cloud are crucial stepping stones between our own Milky Way system and the realm of distant galaxies. In particular the Clouds are the only external galaxies in which it is possible to study both the integrated properties of star clusters and the spectra and colours of

*IAU Symposium No. 108 on Structure and Evolution of the Magellanic Clouds was held in Tithingen, W. Ciermany 5-8 Magellanic Clouds
Scptember, 1983. individual cluster stars. Recent multicolour observations by L. Searle (Mt. Wilson Observatory, Pasadena) are able to order Cloud clusters by relative age while colour-magnitude diagrams of stars within some of these clusters provide an absolute age calibration. Such work has revealed striking differences between the evolutionary histories of the Large Magellanic Cloud and Small Magellanic Cloud on the one hand and the Galaxy on the other.

Exceedingly old clusters, similar to galactic globular clusters, are found to be relatively rare indicating that star and cluster formation got off to a rather slow start in the Magellanic Clouds, contrasting with our Galaxy in which an enormous burst of star and cluster formation seems to have taken place as the Milky Way system collapsed from its gaseous proto-galactic state. In the Galaxy this initial burst of star formation resulted in the subsequent production of vast numbers of supernovae, which rapidly built up the heavy element abundance in the galactic interstellar gas. The Magellanic Clouds seem to have collapsed more slowly; probably because 